\title{
XVI. EXPERIMENTAL PRODUCTION OF PLAGUE EPIDEMICS AMONG ANIMALS.
}

\section{(Second Communication.)}

In the first report (vol. vI. p. 450) we described several series of experiments which had as their object the determination of the relative importance of the Indian rat flea, Pulex cheopis, and of actual close contact in the absence of fleas in the dissemination of plague from animal to animal. These observations were carried out in a series of small godowns or cabins, which were built especially for the purpose. The experiments which we have now to put on record are a continuation of those already published. They were carried out during the non-plague season of 1906 and the plague season of 1907 .

In the first paper we described in detail the structure of the godowns, and pointed out in what way they differ from one another. We need, therefore, now only recall to the reader's memory that the essential difference between them lies in the structure of the roofs. In the case of Nos. 1 and 2 the roofs, being of country tiles, offer good protection and shelter to the wild rat of Bombay; the flea supply in the interior is, therefore, abundant and regular. In the case of godowns 3 and 4 the roofs, being of flat Mangalore tiles, offer only poor protection to rats, and in consequence the flea supply is more or less scanty. As regards Nos. 5 and 6 , it will be remembered that the roofs during the earlier experiments were made of corrugated iron fixed by cement to the tops of the walls. It was found impossible to keep these godowns absolutely free from fleas, and during one experiment, owing either to breeding or to a sudden migration from without through a flaw in the cement, a fairly abundant supply of these insects obtained access to godown No. 6 .

In order to prevent any such unlooked-for circumstance happening again the corrugated iron was removed and a roof of "reinforced concrete" was put on each of these godowns before the present ex- 
periments were begun. By this means we were able to keep Nos. 5 and 6 godowns absolutely free from fleas,.and in consequence to obtain a number of clean contact experiments in which the presence of fleas was rigorously excluded.

\section{Series A.}

Experiments in which epidemic plague did not occur when healthy guinea-pigs lived in close contact under conditions where access of fleas was completely prevented, but in which under otherwise similar conditions plague spread among the healthy animals in places where fleas were abundantly present.

The same methods were adopted in this series of experiments as were used in the similar series already described in the first report. The godowns were not cleaned out during the whole period of the experiment, and the animals took their food from the floor which was contaminated with the excreta of their sick companions. Under these circumstances, as will be seen from the records below, in the case of godowns 5 and 6 , which were free from fleas, no animals ever contracted plague, while in the case of Nos. 1 and 2 godowns, in which fleas were present, plague became epidemic, or not, according to the season of the year at which the experiment was made.

It will be convenient, and will conduce to the better understanding of the subject, if we describe the experiments in pairs, one in a fleafree godown, the other in a godown in which fleas were present. The experiments were always carried out contemporaneously in this way.

\section{Experiment $I$.}

This experiment was carried out in godowns Nos. 6 and 1 during the month of June, 1906, the beginning of the non-epidemic season in Bombay. The minimum temperature in the godowns was never below $80.5^{\circ} \mathrm{F}$. and was frequently above $82^{\circ} \mathrm{F}$.

Godown 6. On 5. VI. 06 there were placed in this godown 25 healthy guinea-pigs, and five guinea-pigs which had been inoculated with a virulent culture of $B$. pestis. By 11. vI. 06 all the inoculated guinea-pigs had died of plague. The uninoculated animals remained healthy until the experiment was ended on 2. vII. 06 . No fleas were ever found on the guinea-pigs during the course of the experiment.

Godown 1. On 5. vi. 06 five guinea-pigs which had been inoculated with a virulent culture of $B$. pestis were placed in the 


\section{Reports on Plague Investigations in India}

godown. A flea census on this day gave 39 fleas caught on five animals. All these guinea-pigs had died of plague by the 10 . vi. 06 . On 10. vi. 06, after the last inoculated guinea-pig had died, 25 healthy guinea-pigs were placed in the godown. On 21. vi. 06 one guinea-pig was found dead. It was proved not to be plague infected. All the other guinea-pigs remained healthy until the experiment was closed on 2. vIr. 06. A flea census made on 19. vi. 06 showed 31 fleas on .25 guinea-pigs, and another count made on 1. vII. 06 gave 56 fleas on 24, guinea-pigs.

\section{Experiment $I I$.}

This experiment was carried out in godowns 6 and 1 during the month of July, 1906, that is, in the non-epidemic season in Bombay. The average mean temperature in the godowns during the period of the experiment was $82 \cdot 3^{\circ} \mathrm{F}$.

Godown 6. On 2. viI. 06 there were placed in this godown 25 healthy guinea-pigs and five others which had been inoculated with a virulent culture of B. pestis. By 6 . vII. 06 all the inoculated guinea-pigs had died of plague. The uninoculated animals all remained well until the experiment was ended on 29. viI. 06. No fleas were ever found on the guinea-pigs during the experiment.

Godown 1. On 2. VII. 06 a preliminary flea count gave 50 fleas on 25 guinea-pigs. On this date there were put into the godown five guinea-pigs which had been inoculated with a virulent culture of $B$. pestis. The last of these animals died of plague on 6. VII. 06 . On 6. vII. 06, after the last inoculated guinea-pig had died, there were placed in the godown 25 healthy guinea-pigs. Between 10 . vil. 06 and 13. vII. 06 four of these healthy animals died, two from plague and two from some other cause. All the other guinea-pigs remained healthy until the experiment was abandoned on 27. vir. 06 .

\section{Experiment $I I I$.}

This experiment was carried out in godowns Nos. 6 and 2 during the month of August, 1906, when plague was not epidemic in Bombay, but at a season of the year when a slight recrudescence has been observed in former years. The mean temperature in the godowns during the period of the experiment was $81 \cdot 1^{\circ} \mathrm{F}$, while the minimum remained about $80^{\circ} \mathrm{F}$.

Godown 6. On 1. VIII. 06 there were placed in this godown 25 healthy guinea-pigs and five which had been inoculated with a 
virulent culture of $B$. pestis. The first inoculated animal died on 6. vIII. 06 and the last on 12 . viII. 06 . The uninoculated animals remained healthy until the experiment was abandoned on 5. IX. 06 . No fleas were taken on the guinea-pigs during the course of the experiment.

Godown 2. On 1. viII. 06, 126 fleas were taken on 25 guineapigs in the godown. The fleas were returned to the godown. On 1. viII. 06 there were placed in the godown five guinea-pigs which had been inoculated with a virulent culture of $B$. pestis. By 8 . viII. 06 all these guinea-pigs had died of plague. On 8. virr. 06, after the last inoculated animal had died, 25 healthy guinea-pigs were placed in the godown. On 15. virr. 06 the first uninoculated guinea-pig died, and by 4. IX. 06 twenty-four out of the twenty-five had died, all from plague. On 5. Ix. 06 on the surviving guinea-pig which appeared to be healthy 88 fleas were taken. The animal was then isolated, but remained healthy.

\section{Experiment $I V$.}

This experiment was carried out in godowns 6 and 2 during the months of September and October, when plague was still non-epidemic in Bombay. The mean temperature in the godowns during the period of the experiment was $81 \cdot 8^{\circ} \mathrm{F}$.

Godown 6. On 21. Ix. 06 there were placed in this godown 25 healthy guinea-pigs and five guinea-pigs which had been inoculated with a virulent culture of $B$. pestis. By 29. Ix. 06 all the inoculated guinea-pigs had died of plague. After this date fresh plague-inoculated guinea-pigs were added to the godown almost daily, the same number of infected animals being put in as died of plague in godown 2 during the course of the present experiment. Thus, between 2. x. 06 and 16. x. 06, twenty-three plague-infected guinea-pigs were added. All these animals died of plague, the last on 19. x. 06. It will be seen, therefore, that between 21. Ix. 06 and 19. x. 06 the twenty-five healthy guinea-pigs were living in close contact with plague-infected animals. On 19. x. 06 one of the 25 uninoculated guinea-pigs was found dead. It was proved not to have died from plague. The remaining 24 animals remained healthy until the experiment was abandoned on $30 . x .06$. No fleas were taken on any of the guinea-pigs during the course of the experiment.

Godown 2. On 21. Ix. 06 there were placed in this godown five guinea-pigs which had been inoculated with a virulent culture 


\section{Reports on Plague Investigations in India}

of B. pestis. All these guinea-pigs died of plague, the last on 27. Ix. 06 . Sixteen fleas which were taken from this animal were returned to the godown. On 27. Ix. 06, after the last inoculated guinea-pig had died, 25 healthy guinea-pigs were put into the godown. On 1. x. 06 five of these animals were found dead, and by 16. x. 06 there only remained two alive. All the deaths were proved to be due to plague. On 17. $x .06$ it was noticed that the two survivors had submaxillary buboes. On $30 . \times 106$ they were seen to be well. On them 101 fleas were taken. The guinea-pigs remained well until the experiment was abandoned on $30 . x .06$.

\section{Experiment $V$.}

This experiment was carried out in godowns 5 and 1 during October and November, 1906, at a season of the year when plague was still nonepidemic in Bombay, but when the rat epizootic was just beginning. The mean temperature in the godowns during the period of the experiment was $80.6^{\circ} \mathrm{F}$.

Godown 5. On 18. x. 06 there were placed in this godown 25 healthy guinea-pigs and five guinea-pigs which had been inoculated with a virulent culture of $B$. pestis. By $23 . \times$ x. 06 all the inoculated guinea-pigs had died of plague. From this date onwards, as in the previous experiment, there were daily added to the godown more plagueinfected animals corresponding to the number which died in godown 1 . Thus between 24. x. 06 and 10. XI. 06 twenty-three plague-infected guinea-pigs were added, all of which died of plague, the last on 15. XI. 06. On some days the "concentration" of infection was very great. Thus, on 28. x. 06 the 25 healthy animals were living in contact with 19 guinea-pigs which were plague-infected. In spite of being thus exposed to severe contact infection for a period of about a month, all the 25 uninoculated guinea-pigs remained healthy until the experiment was abandoned on 28. xI. 06. No fleas were taken on any of these guinea-pigs during the course of the experiment.

Godown 1. On 18. x. 06 four guinea-pigs, which had been in the godown overnight, yielded 546 fleas. On 18. x. 06 there were placed in the godown five guinea-pigs which had been inoculated with a virulent culture of $B$. pestis. All these animals died of plague, the last on 21. x. 06. On 21. x. 06, after the last inoculated guinea-pig had died, 25 healthy guinea-pigs were put into the godown. Plague at once broke out amongst these animals, the first dying on 23. x. 06, and by 10. XI. 06 twenty-three of them had succumbed to the disease. 
On 20. xI. 06 the two animals which remained were examined for fleas, and 416 were taken. They remained healthy till they were returned to stock on 3. XII. 06 . The 416 fleas were put on a fresh guinea-pig in a flea-proof cage in the laboratory. This guinea-pig also remained healthy. It is evident, therefore, that although fleas were abundant the infection had died out.

\section{Experiment VI.}

This experiment was made in godowns 5 and 2 during the month of January, 1907, that is to say, at a season when the plague epizootic in Bombay was rapidly increasing. The mean temperature in the godowns during the period of the experiment was $728^{\circ} \mathrm{F}$., and the maximum was never above $78^{\circ} \mathrm{F}$.

Godown 5. On 8. I. 07 there were placed in this godown 25 healthy guinea-pigs and five guinea-pigs which had been inoculated with a virulent culture of $B$. pestis. By 11. I. 07 all the inoculated animals had died of plague. After this date, as in the two previous experiments, more plague-infected guinea-pigs were added daily, 25 in all being put in between 15. I. 07 and 24. 1. 07. All these guineapigs died of plague, the last death being on 31. I. 07. It is to be noted that on 18. I. 07 there were 21 plague-infected animals in contact with the 25 uninoculated guinea-pigs. These latter, however, remained healthy until the experiment was abandoned on 21. II. 07. No Heas were observed on the guinea-pigs throughout the course of the experiment.

Godown 2. On 8. I. 07 a preliminary flea count yielded 107 fleas on three guinea-pigs. On 8. I. 07 five guinea-pigs which had been inoculated with a virulent culture of $B$. pestis were placed in the godown. By 11. I. 07 all these animals had died from plague. On 11. I. 07, after the last inoculated guinea-pig had died, 25 healthy guinea-pigs were put into the godown. Plague at once broke out amongst these animals, the first dying on 15. I. 07, and in a fortnight from their being placed in the godown the last one had succumbed to the disease.

\section{Summary and Conclusions.}

In six contact experiments, carried out at different seasons of the year in godowns which were absolutely free from fleas, not a single healthy guinea-pig died of plague. In three of these experiments the contact with infected animals was kept up by the daily addition 
of plague-infected animals for a period of from 14 to 21 days, and still none of the healthy animals developed plague. As the godowns were never cleaned out, close contact includes contact with faeces and urine of infected animals, and eating of food contaminated with faeces and urine of infected animals. We can, therefore, conclude that close contact of infected animals with healthy animals, if fleas are excluded, does not give rise to an epizootic amongst the latter.

At the same time as these flea-free contact experiments were being carried out, experiments in similar godowns, but which received a regular supply of fleas from the roofs, were made. The details of these experiments are summarised in the following table. From this table it is seen that the experiment in June failed completely, that the one in July nearly completely failed, and that the four experiments made between August and January were successful. We can conclude that fleas, and fleas only, were responsible for these epizootics. In the January

TABLE.

\begin{tabular}{|c|c|c|c|c|c|c|c|c|c|c|c|}
\hline \multirow[b]{2}{*}{$\begin{array}{l}\text { No.of } \\
\text { Exp. }\end{array}$} & \multirow[b]{2}{*}{$\begin{array}{l}\text { Go- } \\
\text { down }\end{array}$} & \multirow[b]{2}{*}{$\begin{array}{l}\text { Season } \\
\text { of year }\end{array}$} & \multirow[b]{2}{*}{$\begin{array}{l}\text { Average dally } \\
\text { mean tempersture } \\
\text { of godown }\end{array}$} & \multirow[b]{2}{*}{ Flea census } & \multirow{2}{*}{ 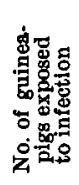 } & \multicolumn{5}{|c|}{$\begin{array}{l}\text { No. of guines-pigs which } \\
\text { died from plague }\end{array}$} & \multirow{2}{*}{ 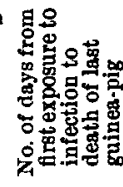 } \\
\hline & & & & & & 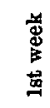 & בั้ & 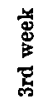 & 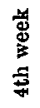 & 馬 & \\
\hline $\mathbf{I}$ & 1 & June 1906 & $\begin{array}{l}\text { Min. temp. never } \\
\text { below } 80.5^{\circ} \mathrm{F} \text {. and } \\
\text { often above } 82^{\circ} \mathrm{F} \text {. }\end{array}$ & $\begin{array}{l}39 \text { on } \\
5 \text { guines-pigs }\end{array}$ & 25 & 0 & 0 & 0 & 0 & 0 & - \\
\hline II & 1 & July 1906 & $82 \cdot 3^{\circ} \mathrm{E}$. & $\begin{array}{c}50 \text { on } \\
25 \text { guinea-pigs }\end{array}$ & 25 & 1 & 1 & 0 & 0 & 2 & 8 \\
\hline III & 2 & Angust 1906 & $81 \cdot 1^{\circ} \mathrm{F}$ & $\begin{array}{c}126 \text { on } \\
25 \text { guinea-pigs }\end{array}$ & 25 & 0 & 14 & 8 & 2 & 24 & 28 \\
\hline IV & 2 & $\begin{array}{c}\text { Sept. \& Oct. } \\
1906\end{array}$ & $81 \cdot 8^{\circ} \mathrm{F}$ & $\begin{array}{l}101 \text { on the last } \\
\text { two guinea-pigs }\end{array}$ & 25 & 7 & 13 & $\mathbf{3}$ & 0 & 23 & 20 \\
\hline $\mathbf{V}$ & 1 & $\begin{array}{c}\text { Oet. \& Nov. } \\
1906\end{array}$ & $80 \cdot 6^{\circ} \mathrm{F}$ & $\begin{array}{l}546 \text { on } \\
4 \text { guinea-pigs }\end{array}$ & 25 & 17 & 4 & 2 & 0 & $\mathbf{2 3}$ & 21 \\
\hline VI & 2 & $\begin{array}{l}\text { January } \\
1907\end{array}$ & $72.8^{\circ} \mathrm{F}$ & $\begin{array}{l}107 \text { on } \\
3 \text { guinea-pigs }\end{array}$ & 25 & 14 & 11 & 0 & 0 & 25 & 14 \\
\hline
\end{tabular}

experiment, that is to say, at a season of the year when the rat epizootic in Bombay city is rapidly rising, the epizootic among the godown guinea-pigs ran a very rapid course, all the animals succumbing to the disease in a comparatively short time. On the other hand, in the three positive experiments during the non-epizootic season in Bombay, in every instance, and especially in August, the epizootic 
among the godown guinea-pigs was more or less drawn out, and one or two of the animals escaped, the infection dying out towards the end.

The bearing of these facts on the seasonal prevalence of plague will be dealt with in another paper, when the factors of the flea prevalence and climatic conditions in relation to plague epidemics will be fully discussed.

\section{Series B.}

Experiments indicating that the number of fleas in the godowns affects the rate of progress of the epizootic amongst the guinea-pigs.

In the previous report we brought forward evidence which indicated that if fleas are present, then the epizootic once started spreads from animal to animal, the rate of progress being directly influenced by the number of fleas present. We pointed out that in the experiments during the months of December, January, and February the progress of the epizootic was very rapid in those godowns, namely, Nos. 1 and 2, in which the flea population was abundant and was kept up by a natural supply from the roof; that it was much slower in godown No. 5 in which the flea supply was kept up artificially; and finally that it was slowest of all in godown No. 6 in which there was no definite natural supply of fleas, and from which the fleas were daily removed for a period of six days, after which removal only a comparatively small number could be caught.

We have now to detail two observations which were made in godowns 3 and 4 which are roofed with Mangalore tiles, and in which the flea infestation has never been so great as in Nos. 1 and 2 (vide p. 453 of last report). We shall contrast the rate of progress of the epizootics in these godowns with its rate in godowns 1 and 2 at the same season of the year, that is to say, when the climatic conditions were suitable.

\section{Experiment VII.}

Godown 4. On 5. XII. 05 twenty healthy guinea-pigs were placed in this godown. On 6. XII. 05 a flea census was made and 35 fleas were taken on 12 animals. Between this date and 13. XII. 05 somehow or other plague infection got into the godown, the first guinea-pig dying on 13. XII. 05. The epizootic once started went slowly through the rest of the guinea-pigs, the last animal dying on 15. I. 06, that is to say, it took 33 days to kill all the 20 guinea-pigs. This experiment 
can be contrasted with an experiment which was made in godown 1 at the same time (Experiment V, p. 457 of previous report). In this instance only seven days elapsed between the death of the first and last guinea-pigs, although the epizootic had to pass through 26 instead of 20 animals. In this case the fleas were present in very large numbers, 115 being taken on the last five animals when moribund.

\section{Experiment VIII.}

Godowns 4 and 2. This experiment was carried out in godowns 4 and 2 during the rise and height of the plague epizootic of 1907.

Godown 4. On 29. I. 07 a flea census yielded nine fleas on three guinea-pigs. On 30. I. 07,100 fleas were added. On 1. II. 07 five guinea-pigs which had been inoculated with a virulent culture of $B$. pestis were put into the godown. The first of these animals died on 3. II. 07, and the last on 5. II. 07. On 5. II. 07, after the last inoculated guinea-pig had died, 25 healthy guinea-pigs were allowed to run about free in the godown. The course and rate of progress of the epizootic which broke out amongst these animals are well shown in the accompanying chart (p. 430). The first animal died on 12. II. 07 and after that date the epizootic progressed extremely slow, the last guinea-pig dying on 14. Iv. 07. From the date of the exposure of the guinea-pigs to infection until the death of this last animal there was therefore an interval of 68 days. A census of the fleas in the godown was made on several occasions during the course of the experiment. The results of these counts are recorded on the chart. It will be seen that except at the end of the experiment, when only the last animal remained alive, the fleas were at no time numerous. The large increase towards the end was probably due to breeding, . which for some reason or other suddenly started. In great contrast with the epizootic of slow progress in this godown is the epizootic which took place in godown 2, while the former was in progress.

Godown 2. On 1. III. 07 a census yielded 1246 fleas caught on one guinea-pig. On 1. III. 07 five guinea-pigs which had been inoculated with a virulent culture of $B$. pestis were put into the godown. The first of these animals died on 5. III. 07 and the last on 7. III. 07 . On 6. III. 07, while there was still one of the inoculated guinea-pigs alive, 25 healthy guinea-pigs were allowed to run free in the godown. On 9. III. 07 the first uninoculated guinea-pig died; on 10. III. 07 sixteen died; on 11. III. 07 four; on 12 . III. 07 four; 
all of these animals were proved to be plague-infected. On 12. III. 07, that is to say, six days after the guinea-pigs had been exposed to infection, only one remained alive. This guinea-pig was noticed to be sick and to have a bubo in the cervical region. On 15. III. 07,

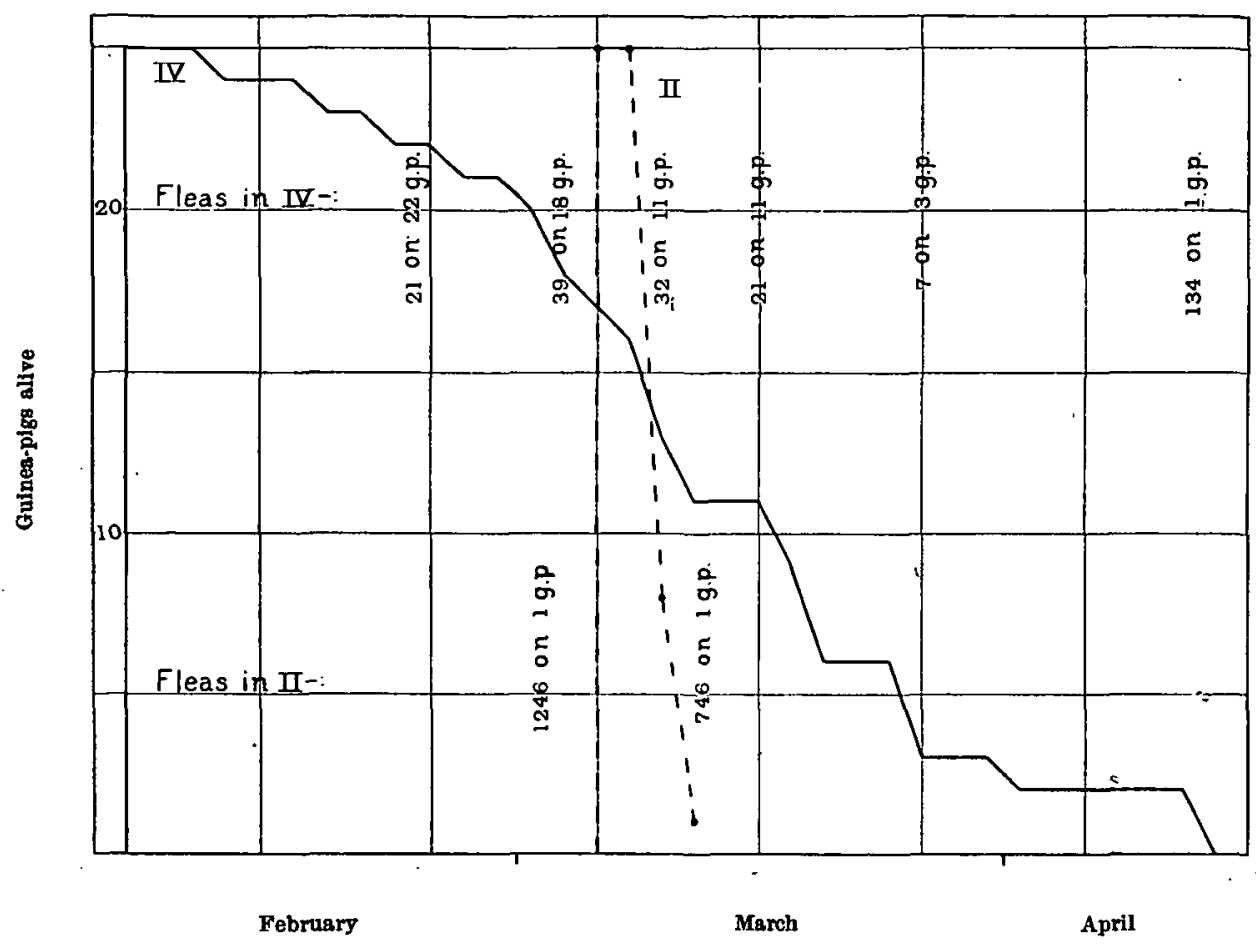

Chart showing the rate of progress of the epizootics which occurred at the same season of the year, one in godown 4 (black line) and the other in godown 2 (broken line). The curves are constructed on the number of guinea-pigs left alive in the godowus at intervals of two days.

as it appeared to be recovering, it was killed with chloroform and examined bacteriologically. A pure culture of $B$. pestis was obtained. The rate of progress of this epizootic is shown as a broken line on the chart. Its great rapidity as contrasted with the slowness of the epizootic in No. 4 godown is very remarkable. As both experiments were made at the same season of the year and under the same conditions, except as regards the number of fleas present, we can only conclude that this latter factor was the one which determined the difference between the two epizootics. 


\section{Summary and Conclusions.}

Two experiments are cited, in each of which the plague epizootic amongst the guinea-pigs in a godown, which was only slightly infested with fleas, is contrasted, as regards its rate of progress, with that amongst the guinea-pigs in a godown which was abundantly supplied with fleas. In each instance the rate of progress is shown to be much slower in the former than in the latter godown. We have, therefore, confirmation of our previous conclusion that, if fleas are present, then the epizootic once started spreads from animal to animal, the rate of progress being conditioned by the number of fleas present. The question of the influence of a flea prevalence on the seasonal prevalence of plague will be dealt with in another paper.

\section{SERIES C.}

Experiment indicating that, when an epidemic has occurred amongst a number of guinea-pigs, the contagion still remains in the place, and is effective in proportion as the test animals are accessible to, and found to be infested with, fleas.

The single experiment which makes up this series is a confirmation of Experiments VII and IX of Series C described in the last report.

\section{Experiment IX.}

On 28. I. 07 into godown 2, in which a plague epizootic had just come to an end by killing all the guinea-pigs, three groups of four guinea-pigs each were placed, namely,

$(A)$ Four guinea-pigs running about free on the floor;

(B) Four guinea-pigs in wire cages two inches above the ground; and

(C) Four guinea-pigs in wire cages suspended two feet above the ground.

On 30. I. 07 these guinea-pigs were freed from fleas under chloroform, removed from the godown, and segregated. 125 fleas were caught on the four guinea-pigs which had been running about, and 27 on the four which had been placed in cages two inches from the ground. No fleas were taken on the animals which had been suspended two feet above the ground. The eight animals of groups $A$ and $B$ were observed to be ill and all to have buboes in the cervical region. Three showed typical phlyctenules under their chins. The 
four guinea-pigs of group $C$ appeared quite well. The fate of the animals of these three groups was as follows:-

Group $A$. One died under chloroform while the fleas were being removed. It was proved bacteriologically to be plague infected, the bubo being in the cervical region. The other three guinea-pigs all died of plague, one on 31. I. 07, one on 1. II. 07, and the third on 2. II. 07. The buboes were all cervical.

Group $B$. All four animals died of plague, three on 31. I. 07, and the fourth on 2. II. 07. The bubo was in every instance in the cervical region.

Group C. All four guinea-pigs remained healthy.

\section{Conclusions.}

Infection can therefore take place without any contact with contaminated soil, as the guinea-pigs which were kept two inches above the ground all contracted the disease. Aerial infection is also excluded, since the guinea-pigs which were suspended two feet from the ground remained healthy, while those running free and those two inches from the ground became infected.

We conclude that infected fleas were the agents of transmission in this experiment.

\section{Series D.}

Experiments in which monkeys, protected and unprotected from fleas, were exposed in an infected godown. The protected monkeys never developed plague, while a certain number of those left unprotected were attacked.

The cages which were used in this series of experiments were of the same pattern as those employed in our experiments in plagueinfected houses in Bombay. They have already been described and figured (vide Plates VI and VII, p. 476 of first report). The essential feature was that in one cage protection from fleas was obtained by means of a layer of fine-meshed metallic gauze, which was absent from the unprotected cages.

\section{Experiment $X$.}

Godown 1. On 23. XI. 06 a preliminary flea count yielded 682 fleas on three guinea-pigs. On this day three guinea-pigs which had been inoculated with a virulent culture of $B$. pestis were placed in the 
godown. These three guinea-pigs had all died of plague by 30 . XI. 06 . On 30. XI. 06, after the death of the last guinea-pig, four monkeys were put into the godown, two protected, and two unprotected from fleas. On 2. XII. 06 the cages were removed, the monkeys freed from fleas under chloroform, and isolated. No fleas were got on the monkeys which had been in the wire gauze cages, while 16 fleas were got on one of the unprotected animals and 48 on the other. Both the protected monkeys remained well, while both the unprotected died of plague, the one on which the greater number of fleas was taken dying five days before the other. The diagnosis was bacteriologically confirmed in each instance. On 3. XII. 06, that is the day after the four monkeys were removed from the godown, without any fresh infection being introduced, two monkeys in ordinary open cages were put into the godown. On 5. XII. 06 the monkeys were removed, cleared of fleas under chloroform, and isolated. On one animal 23 fleas and on the other 33 fleas were obtained. On 8. xil. 06 the monkey which had the greatest number of fleas on it died of plague. The other monkey remained healthy.

\section{Experiment $X I$.}

Godown 1. On 9. xIr. 06 a preliminary flea count gave 313 fleas on three guinea-pigs. On 10. XII. 06 there were placed in the godown three guinea-pigs which had been inoculated with a virulent culture of $B$. pestis. The last of these animals died on 14. XII. 06. On 14. XII. 06 four monkeys were placed in the godown, two in wire gauze cages, and two in cages which left them unprotected from fleas. On 16. XII. 06 the cages were removed, the monkeys freed from fleas under chloroform, and then isolated. On the protected monkeys no fleas were obtained, while five fleas on one and 21 fleas on the other of the unprotected animals were taken. All four animals remained healthy.

\section{Experiment $X I I$.}

Godown 1. On 12. 1. 07 a preliminary flea count yielded 194 fleas on six guinea-pigs. On this date three guinea-pigs, which had been inoculated with a virulent culture of $B$. pestis, were placed in the godown. By 17. I. 07 all these guinea-pigs had died from plague. On 17. I. 07 four monkeys were placed in the godown, two in wire gauze cages, and two in cages which gave access to fleas. On 19. I. 07 the cages were removed, the monkeys freed from fleas under chloroform, 
and then isolated. On the protected monkeys no fleas were obtained, while 18 fleas on one and 24 fleas on the other of the unprotected animals were taken. All four monkeys remained healthy.

\section{Experiment $X I I I$.}

Godown 1. On 19. I. 07 three guinea-pigs which had been inoculated with a virulent culture of $B$. pestis were placed in the godown. On 20. I. 07 a flea census yielded 271 fleas on these animals. They had all died of plague by 23. I. 07. On 22. I. 07, the last inoculated guineapig being still alive, four monkeys were placed in the godown, two in wire gauze cages, and two in cages which did not give protection from fleas. On 25. I. 07 the cages were removed, the monkeys freed from fleas, and isolated. On the protected animals no fleas were obtained, while 19 fleas from one and two fleas from the other of the unprotected monkeys were taken. Both the protected animals remained well. The monkey on which 19 fleas were obtained died of plague on 27. I. 07. The monkey on which two fleas were got remained healthy.

\section{Experiment $X I V$.}

Godown 2. On 2. II. 07, when an epizootic amongst a number of guinea-pigs was just coming to an end in this godown, the last guineapig not having yet died, four monkeys were placed in the godown, two in wire gauze cages, and two in cages which did not give protection from fleas. On 4. I1. 07 the guinea-pig died, and 36 fleas taken from its corpse were returned to the godown. On 5. II. 07 the monkeys were removed from the godown, freed from fleas, and segregated. On the protected animals no fleas were found, while seven fleas from one and two fleas from the other of the unprotected monkeys were taken. Both the protected animals remained well. The monkey on which two fleas were obtained died of plague on 8. II. 07. The other monkey remained healthy.

\section{Conclusions.}

Five experiments with 22 monkeys were made; in 10 cases they were protected from fleas and in $\mathbf{1 2}$ were unprotected. None of the protected animals contracted the disease, while five out of the 12 unprotected monkeys died of plague.

Soil infection was excluded, and all the monkeys were equally exposed to aerial infection. 
We must conclude, therefore, that infected fleas in the godown were alone responsible for the infection of the five unprotected animals which died of plague.

\section{Summary and Conclusions.}

1. Close and continuous contact of plague-infected animals with healthy animals, if fleas are excluded, does not give rise to an epizootic among the latter. As the godowns were never cleaned out, close contact includes contact with faeces and urine, and eating of food contaminated with faeces and urine of infected animals.

2. When fleas are present, the epizootic, if it does start, varies in severity and rate of progress according to the season of the year and the number of fleas present. The season in which epizootics were readily produced experimentally, and spread rapidly, corresponds with that of the plague epidemic.

3. An epizootic of plague may occur in a godown containing infected fleas without direct contact of healthy animals and infected animals (Experiments III, IV, V, and VI).

4. In an infected godown the infection is effective in proportion as the test animals are accessible to fleas.

5. Infection can take place without any contact with contaminated soil (Experiments IX to XIV).

6. The experiments exclude aerial infection.

7. The experiments lead to the conclusion that fleas, and fleas alone, were the transmitting agents of infection. 